\title{
Phase-field numerical simulation of three- dimensional competitive growth of dendrites in a binary alloy
}

\author{
*Li Feng ${ }^{1,2}$, Ya-long Gao ${ }^{1}$, Chang-sheng Zhu ${ }^{2,3}$, Guo-sheng An ${ }^{1}$, Xin Deng ${ }^{3}$, and Bei-bei Jia ${ }^{1}$ \\ 1. School of Materials and Engineering, Lanzhou University of Technology, Lanzhou 730050, China \\ 2. State Key Laboratory of Advanced Processing and Recycling of Nonferrous Metals, Lanzhou University of Technology, Lanzhou 730050, China \\ 3. School of Computer and Communication, Lanzhou University of Technology, Lanzhou 730050, China
}

\begin{abstract}
The normal vector of migration direction in the solid-liquid interface of dendrites was used to describe the phase-field governing equation. By using the three angles formed by the normal vector for the migration direction of the dendritic growth interface and the coordinate axes of the simulation region, the authors expressed the interfacial anisotropy equation, and built a phase-field model for the competitive growth of multiple grains. Taking a Al-2\%mole-Cu binary alloy as an example, the competitive growth of multiple grains during isothermal solidification was simulated by applying parallel computing techniques. In addition, the phase field simulation results were verified by the experimental method. The simulation results show that the competitive growth of equiaxed dendrite is divided into two types: the first occurs during the process of competitive growth, the tips of primary dendrite on different grains taking part in the competition stop growing in their optimal growth direction; the second also occurs during competitive growth, the tips of primary dendrite which participate in the competition on different grains never stop growing in their optimal growth direction. The dendritic morphologies of the first competition growth type are divided into two types. Primary dendrites of grains taking part in the competition stop growing in their optimal growth direction and the competition plane enlarges when neither one wins the competition. However, when one wins the competition, the primary dendrites of grains with superiority go through the blocking grains and continue to grow in their optimal growth direction. The primary dendrites of inferior grains stop growing in their optimal growth direction and then instead grow in those areas without obstacles. The dendritic morphology of the second competition-growth type is shown to be the deformation of primary dendrites, which are mainly represented as the deflection and bending observed from different views. Compared with the metallographic picture, the simulation results can show the morphology of the competitive growth in all directions, so this simulation method can better characterize the competitive growth process.
\end{abstract}

Key words: dendrite; Al-Cu binary alloy; competitive growth; dendritic morphology

CLC numbers: TP391.99./TG146.21 Document code: A Article ID: 1672-6421(2018)01-044-07

\begin{abstract}
$\Delta$ siel S an important simulation method, the phasefield method (PFM), which enables researchers to directly simulate the formation of microstructures, is an efficient tool for studying the structure of complex solidification interfaces. PFM can couple nucleation and growth models and influencing factors of nucleation and growth process with the basic heat transfer equation to carry out accurate microscopic numerical simulation. In
\end{abstract}

\footnotetext{
* Li Feng

Male, born in 1981, Associate Professor. His research interests mainly focus on the numerical simulation of microstructure in solidification process.

E-mail: fenglils@lut.cn

Received: 2017-04-12; Accepted: 2017-10-11
}

this way, the quantitative relationship of the effect of primary process parameters on the microstructure during material forming processes can be obtained. As an open model, the phase-field model can be coupled with various external fields such as temperature, dissolution, gravity, and fluid fields, and can effectively combine the microscale with the macroscale. With the development of the phase-field model and improvements in computing technology, quantitatively simulating and predicting the microstructure in the phase transition of actual materials by using PFM has become the focus of research into this aspect of material structures ${ }^{[1-8]}$.

Dendrite is a common solidification microstructure. 
During the past decade, simulations of dendritic growth by using PFM have become well developed. Simulation studies including 2-D ${ }^{[9-18]}$ and 3-D dendritic growth ${ }^{[2,19-27]}$, dendritic growth of pure substances ${ }^{[9-11,19-20]}$, dendritic growth in binary alloys ${ }^{[1,12-15,21-25]}$, and dendritic growth in multiple alloys ${ }^{[2,16-}$ ${ }^{18,26-27]}$ have been reported. Multiple dendrites simultaneously grow during the casting process and their growth process can be directly simulated by using PFM ${ }^{[1,28-31]}$. Mutual interactions and competitions exist among grains in the simultaneous growth of multiple grains. However, few studies have been carried out on the competitive growth among dendrites. Souhar and De Felice et al. ${ }^{[32]}$ simulated the mutual influence and competition of multiple 3-D dendrites simultaneously growing in a binary alloy by employing a mesoscopic model for solidification. Compared with PFM, mesoscopic models can readily simulate the mutual influence and competitive growth of 3-D dendrites due to the simplicity of calculation. However, although it entails simple calculations, it loses numerous detailed facets in the data. Takaki and Ohno et al. ${ }^{[33,34]}$ simulated competition growth and the mutual influence of 2-D dendrites in a binary alloy under directional solidification by applying PFM and a parallel numerical computation method. Tourret et al. ${ }^{[35]}$ studied competitive growth of 2-D dendrites in a binary alloy during directional solidification by adopting PFM. Moreover, compared with the experimental data, they considered that the 3-D dendritic morphology plays an important role in dendritic orientation selection.

In this paper, by using PFM and parallel computing techniques, the 3-D competitive growth of multiple dendrites was simulated to reproduce the competitive growth of equiaxed dendrites in different directions. In addition, trends in competitive growth of dendrites were explored, as well as the morphological characteristics of competitive growth of dendrites and the effects thereof on dendritic orientation selection. Furthermore, the phase field simulation results were verified by the experimental method.

\section{Phase-field model of competitive growth of dendrites}

\subsection{Phase-field and solute-field governing equations}

Here, the normal vector in the solid-liquid interface of dendrites in migration direction is defined as follows:

$$
\vec{n}=\frac{\nabla \phi}{|\nabla \phi|}
$$

The phase-field governing equation described in the literature ${ }^{[36]}$ is employed:

$$
\frac{\partial \phi}{\partial t}=M\left(\varepsilon(\theta)^{2} \nabla^{2} \phi+\left(\varepsilon(\theta) \cdot \varepsilon^{\prime \prime}(\theta)+\varepsilon^{\prime}(\theta)^{2}\right) \cdot(|\nabla \phi| \nabla \vec{n})-f_{\phi}\right)
$$

where $\phi, M$, and $t$ represent the order parameter of phase field, the migration rate of solid-phase interface, and the time variable, respectively. Moreover, $\varepsilon(\theta)$ and $f_{\phi}$ refer to the parameter related to the surface energy and the first-order derivative of free energy density with respect to the order parameter of the phase field, respectively. The latter can be expressed as follows:

$$
f_{\phi}=\frac{R T}{V_{m}} h^{\prime}(\phi) \ln \frac{\left(1-c_{\mathrm{S}}^{\mathrm{e}}\right)\left(1-c_{\mathrm{L}}\right)}{\left(1-c_{\mathrm{L}}^{\mathrm{e}}\right)\left(1-c_{\mathrm{S}}\right)}+W g^{\prime}(\phi)
$$

where $R, T, V_{\mathrm{m}}, h(\phi), W, g(\phi)$, and $c$ represent the universal gas constant, temperature, molar volume, potential function, phase-field parameter, residual free energy function, and solute concentration of the alloy, respectively. Moreover, subscripts $\mathrm{L}$ and $\mathrm{S}$ as well as superscript e refer to the liquid phase, solid phase, and an equilibrium state, respectively.

The governing equation describing the diffusion and redistribution of solute can be written as follows:

$$
\frac{\partial c}{\partial t}=\nabla\left(\frac{D(\phi)}{f_{c c}} \nabla f_{c}\right)
$$

where $D(\phi)$ is the solute diffusion rate while $f_{c}$ and $f_{c c}$ represent the first- and second-order derivatives of free energy with respect to concentration, respectively.

\subsection{Function equation for anisotropy of interfacial energy}

There are three angles $\left(\theta_{1}, \theta_{2}\right.$, and $\theta_{3}$, respectively) between the normal direction for the migration direction of the dendritic growth interface and the coordinate system:

$$
\begin{aligned}
& \theta_{1}=\arccos \left(\frac{\phi_{x}}{\sqrt{\left(\phi_{x}\right)^{2}+\left(\phi_{y}\right)^{2}+\left(\phi_{z}\right)^{2}}}\right) \\
& \theta_{2}=\arccos \left(\frac{\phi_{y}}{\sqrt{\left(\phi_{x}\right)^{2}+\left(\phi_{y}\right)^{2}+\left(\phi_{z}\right)^{2}}}\right) \\
& \theta_{3}=\arccos \left(\frac{\phi_{z}}{\sqrt{\left(\phi_{x}\right)^{2}+\left(\phi_{y}\right)^{2}+\left(\phi_{z}\right)^{2}}}\right)
\end{aligned}
$$

where subscripts $x, y$, and $z$ represent the first-order derivatives of the order parameter of the phase field with respect to the spatial coordinates $x, y$, and $z$, respectively. The angle $\theta$ between the normal direction for the migration of the dendritic growth interface and the optimal growth direction can be described by $\theta_{1}, \theta_{2}$, and $\theta_{3}$. Also, by using the $\theta$ as described by the three angles, the function for the anisotropy of interfacial free energy is defined ${ }^{[36]}$. By using $\theta_{1}, \theta_{2}$, and $\theta_{3}$ to define the equation for the anisotropy of interfacial energy, the following equation for the anisotropy of interfacial energy can be obtained:

$$
\varepsilon(\theta)=1-3 \varepsilon_{4}+4 \varepsilon_{4}\left(\cos ^{4} \theta_{1}+\cos ^{4} \theta_{2}+\cos ^{4} \theta_{3}\right)
$$

In this model, $\theta_{1}, \theta_{2}$, and $\theta_{3}$ need to meet the following requirement: 


$$
\cos ^{2} \theta_{1}+\cos ^{2} \theta_{2}+\cos ^{2} \theta_{3}=1
$$

Equations (8) and (9) control the growth orientation of grains, so the growth orientation of grains changes as the initial values of $\theta_{1}, \theta_{2}$, and $\theta_{3}$ vary.

\section{Phase-field parameters and numerical calculation of model}

\subsection{Determination of phase-field parameters}

Migration rate $M$ of solid-phase interface can be written as:

$$
M^{-1}=\frac{\varepsilon_{0}^{3}}{\sigma \sqrt{2 W}}\left(\frac{1}{D} \zeta\left(c_{\mathrm{L}}^{\mathrm{e}}, c_{\mathrm{S}}^{\mathrm{e}}\right)\right)
$$

where $\varepsilon_{0}$ and $W$ are phase-field parameters, which are calculated based on interface energy $\sigma$ and interface thickness $\lambda$. They can be written as:

$$
\begin{aligned}
& \varepsilon_{0}=\sqrt{\frac{6 \lambda \sigma}{2.2}} \\
& W=\frac{6.6 \sigma}{\lambda}
\end{aligned}
$$

$\zeta$ is the interface migration parameter matrix. The expression can be written as:

$$
\begin{aligned}
& \zeta=\frac{R T}{V_{\mathrm{m}}}\left(c_{\mathrm{L}}^{\mathrm{e}}-c_{\mathrm{S}}^{\mathrm{e}}\right)^{2} \\
& \times \int_{0}^{1} \frac{h(\phi)[1-h(\phi)]}{[1-h(\phi)] c_{\mathrm{L}}^{\mathrm{e}}\left(1-c_{\mathrm{L}}^{\mathrm{e}}\right)+h(\phi) c_{\mathrm{S}}^{\mathrm{e}}\left(1-c_{\mathrm{S}}^{\mathrm{e}}\right)} \bullet \frac{d \phi}{\phi(1-\phi)}
\end{aligned}
$$

\begin{tabular}{|c|c|}
\hline Physical parameters & $\mathrm{Al}-2 \%$ moleCu \\
\hline Interfacial energy $\sigma,\left(\mathrm{J} \cdot \mathrm{m}^{-1}\right)$ & 0.093 \\
\hline Melting temperature $T_{\mathrm{m}},(\mathrm{K})$ & 933.3 \\
\hline Equilibrium constant $k^{e}$ & 0.14 \\
\hline $\begin{array}{l}\text { Diffusion coefficient of liquid- } \\
\text { phase solute } D_{\mathrm{L}},\left(\mathrm{m}^{2} \cdot \mathrm{s}^{-1}\right)\end{array}$ & $3.0 \times 10^{-9}$ \\
\hline $\begin{array}{l}\text { Diffusion coefficient of solid- } \\
\text { phase solute } D_{\mathrm{s}},\left(\mathrm{m}^{2} \cdot \mathrm{s}^{-1}\right)\end{array}$ & $3.0 \times 10^{-13}$ \\
\hline Liquidus slope $m^{\ominus},\left(\mathrm{K} \cdot \mathrm{mol}^{-1}\right)$ & 620 \\
\hline Molar volume $V_{\mathrm{m}},\left(\mathrm{m}^{3} \cdot \mathrm{mol}^{-1}\right)$ & $1.0547 \times 10^{-5}$ \\
\hline
\end{tabular}

\subsection{Material physical parameters}

In this work, $\mathrm{Al}-2 \%$ moleCu alloy was used to simulate dendritic growth. The physical parameters of the alloy are listed in Table 1 .

Table 1: Material physical parameters

\subsection{Numerical calculation}

The model was calculated using an MPI (message passing interface). Corresponding to the $x$-, $y$-, and $z$-axes of the rectangular coordinate system, the computational domain of the phase and solute fields was within an area of $400 \times 400 \times 400$ meshes, with a mesh size of $1 \times 10^{-8} \mathrm{~m}\left(\Delta x=1 \times 10^{-8} \mathrm{~m}\right)$.

The initial nucleation was set as a sphere with the radius of the mesh being $R=1 \times 10^{-7} \mathrm{~m}$. By employing an explicit finite difference method, equations (2) and (4) are synchronously solved with the same time-step $\Delta t . \Delta t$ and space-step $\Delta x(\Delta x=$ $\Delta y=\Delta z$ ) need to satisfy the following stable condition:

$$
\Delta t<\min \left\{\Delta x^{2} /\left(6 D_{\mathrm{L}}\right), \Delta y^{2} /\left(6 D_{\mathrm{L}}\right), \Delta z^{2} /\left(6 D_{\mathrm{L}}\right)\right\}
$$

The growth orientation of grains can be controlled by equation (8) and initial values of $\theta_{1}, \theta_{2}$, and $\theta_{3}$.

\section{Results and analysis}

\subsection{Simulation results: morphology of competitive growth of multiple grains and concentrations of solutes}

Figure 1 shows the simulation result for the competitive growth of four grains within the $400 \times 400 \times 400$ mesh. Figures 1 (a1-a6) show 3-D simulation results at various times, and the coordinate axes are included in the upper part of each figure. Figures 1(a1a2), 1(a3-a4), and 1(a5-a6) show the simulation results at

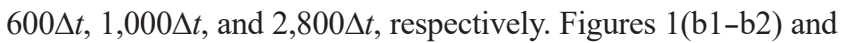
1(c1-c2) show the projections of the section in Fig. 1(a2) on the xoz-plane while the coordinates in Figs. 1(b1-b2) indicate the coordinate plane of Figs. 1(b1-b2) and 1(c1-c2). Figures 1(b3b4) and 1(c3-c4) are projections of the section in Fig. 1(a4) on the yoz-plane while the coordinates in Figs. 1(b3-b4) represent the coordinate planes of Figs. 1(b3-b4) and 1(c3-c4). Figures 1(b5-b7) and 1(c5-c7) are the projections of the section in Fig. 1(a6) on the yoz-plane while the coordinates in Figs. 1(b5-b7) indicate the coordinate planes of Figs. 1(b5-b7) and 1(c5-c7). Figures 1(b1-b7) show the phase-field distribution of sections in the corresponding spaces while Figs. 1(c1-c7) indicate the distribution of the solute field of sections in the corresponding spaces. The upper colour scales in Fig. 1 show the order parameter of the phase field in the corresponding Figs. 1(b1b7), while the bottom colour scales represent the scales of the solute content in the corresponding Figs. 1(c1-c7).

At $600 \Delta t$, the four grains separately grow and reflect their own anisotropy on the interface without mutual interaction, as shown in Figs. 1(a1-a2), 1(b1-b2), and 1(c1-c2). At 1,000 $\Delta t$, two grains begin to touch each other while the other two do not begin to affect each other, as shown in Figs. 1(a3-a4), 1(b3-b4), and $1(\mathrm{c} 3-\mathrm{c} 4)$. At 2,800 $\Delta$, the four grains show competition for growth to form a complex grain boundary, as illustrated in Figs. 1(a5-a6), 1(b5-b7), and 1(c5-c7). During the growth process, alloy grains release solute atoms which exert an effect on the distribution of the solute of surrounding liquid alloys, as shown in Figs. 1(c1-c7). The area between the two grains is mutually affected by the solute atoms released by the surrounding two grains, and, therefore, shows a high solute concentration, as shown in Figs. 1(c5-c7). According to the colour scale of solutes shown in the figure, the roots of primary branches of the dendrites also exhibit high solute concentrations. In the liquid metal area affected by multiple grains, the competitive growth of grains changes the morphology of the dendrites to form the grain boundaries with complex structures because of mutual competitive growth of grains. 


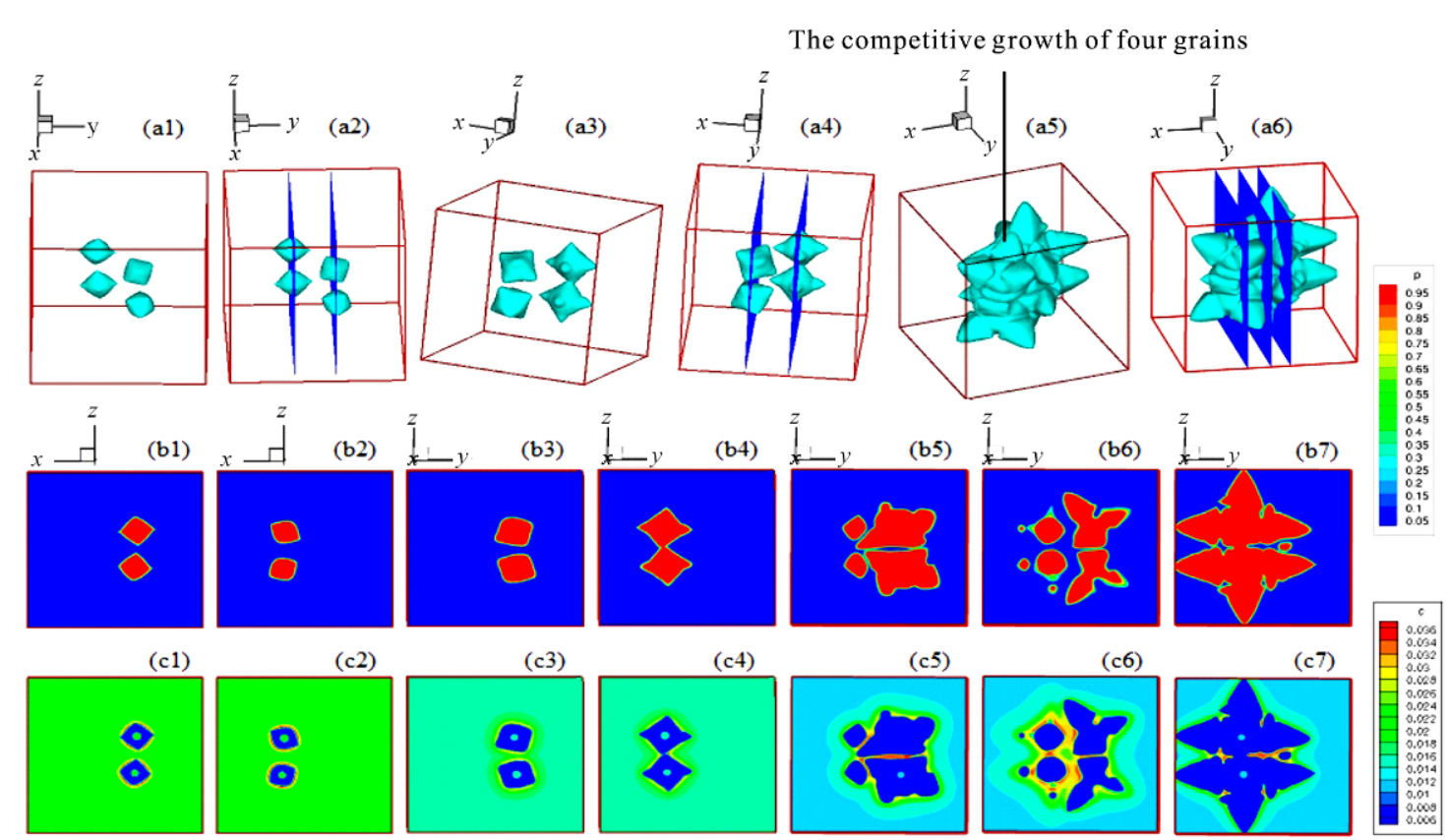

Fig. 1: Simulation results and sectional drawings at different times in competitive growth of multiple grains

To observe the morphologies of each grain competition for growth and to summarise the trends influencing competitive growth on the morphology of the grains, Figure 2 shows the morphologies of all grains corresponding to Fig. 1(a5). Figure 2(a1) shows a 3-D image of Fig. 1(a5) observed from the view of the $x y z-o$ plane and the relevant view coordinates are shown as the coordinates in the top left-hand corner of Fig. 2. Figures 2(a2-a5) show 3-D images of the four grains observed from the same view. Figures 2(b1-b5) show Figs. 2(a1-a5) on the yox-plane while Figs. 2(c1-c5) show Figs. 2(a1-a5) on the zox-plane. Moreover, Figures 2(d1-d5) show Figs. 2(a1-a5) on the zoy-plane. In Fig. 2, the grains in Fig. 2(a2) make contact with those in Fig. 2(a4) and their preferred growth orientations are similar. Therefore, the grain boundaries between them can be classified as low-angle boundaries. However, the grains in Fig. 2(a3) make contact with those in Fig. 2(a5) showing a significant difference in preferred growth orientations, so the grain boundaries between them can be classified as high-angle boundaries. In addition, at $2,800 \Delta t$, no grain boundary was seen between each pair of contacting grains. As can be seen from Fig. 2, although non-contacting dendrites have no crystal boundary, they still influence each other, which causes deformation of the grain morphology, e.g., the deformation of the upper-, and rightbranches of the grains shown in Fig. 2(b2).

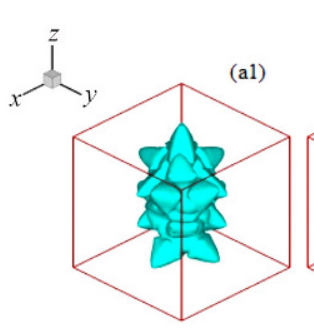

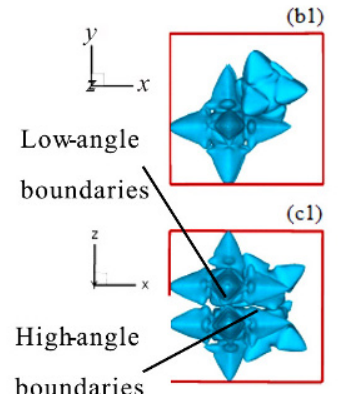

boundaries (d1)

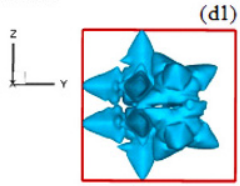

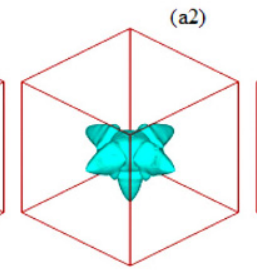

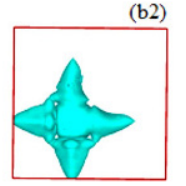

(c2)
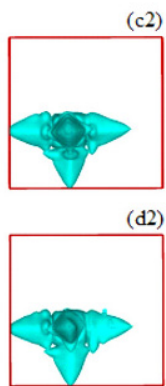

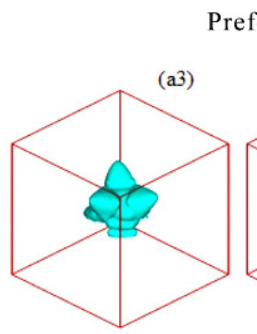

(b3)

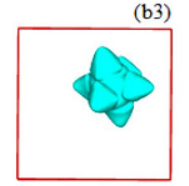

(c3)
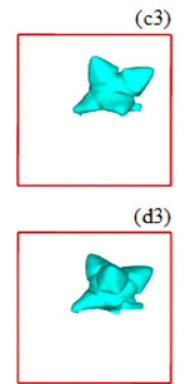
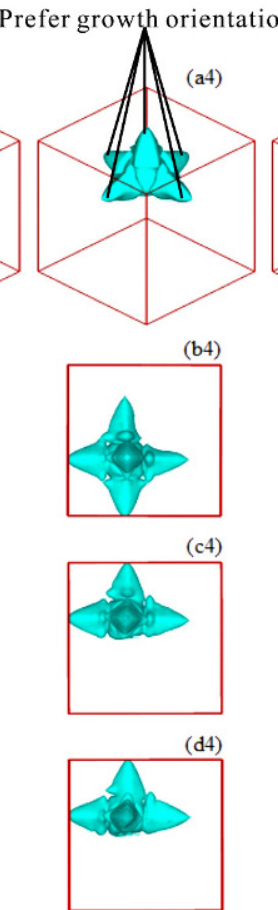

(a5)
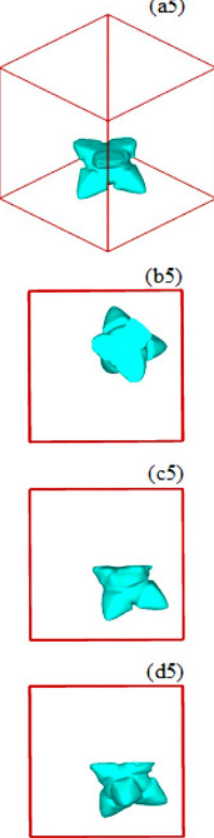

Fig. 2: Simulation of morphologies during competitive growth of multiple grains and single grains (different views) 


\subsection{Analysis and discussion of competitive growth of multiple grains}

According to the simulation results, the mutual influence among grains produced during competitive growth of multiple grains can be divided into two types: (1) during the competitive growth, the tips of the primary branches of those dendrites participating in the competition on different grains stop growing in their optimal growth direction; (2) during competitive growth, the tips of the primary branches of the dendrites taking part in the competition do not stop growing in their optimal growth direction.

In Figs. 2(a2) and (a4), the competition of grains belongs to the first type: at $600 \Delta t$, the upper primary branch of the grain in Fig. 2(a2) has no contact with any other or the lower primary branch of the grain in Fig. 2(a4), as shown in Figs. 1(a1-a2) and $1(\mathrm{~b} 1-\mathrm{b} 2)$. In time, the tips present increasingly narrow separations and finally make contact with each other, as shown in Figs. 1(a3) and 1(b4). Finally, the primary branches of the two dendrites stop growing in the direction (parallel to the $z$-axis) after making contact, as shown in Figs. 1(b7) and 2(c1). The competitive growth of grains in Figs. 2(a3) and 2(a5) also belongs to this type, but it displays certain differences therewith. The optimal growth direction of the primary dendrites of grains in Figs. 2(a2) and 2(a4) was at a low angle, while those in Figs. 2(a3) and 2(a5) grow at a steeper angle. The details of their differences are described below.

The competition of grains in Figs. 2(a3) and 2(a4) is of the second type, as shown in Figs. 2(b1), 2(b3), and 2(b4). The lower primary branch of the grain in Fig. 2(b3) interacts with the right primary branch of the grain in Fig. 2(b4), resulting in a morphological deformation of the primary branches. Competition between grains in Figs. 2(a3) and 2(a4) also belongs to this type, as shown in Figs. 2(d1), 2(d3), and 2(d5). The morphology of the primary branch of the grain pointing outward (parallel to the $x$-axis) in Figs. 2(d2) and 2(d4) deflects. The deflection is performed as the deformation of the right primary branch of the grain seen in Figs. 2 (b2) and 2 (b4) from the perspective of the $x$-axis.

As mentioned above, in the competitive growth of the first type, different grains having large or small angles between their optimal growth directions show different morphologies. Figure 3 shows the morphologies of dendrites competing for growth forming a large angle with the optimal growth directions. Figures 3(a1-a5) show the morphologies of the two grains observed from different views and the coordinate axes above the figures correspond to the direction of the view. Figures 3(b1-b5) display the morphologies of one grain winning the competition observed from different views and the coordinate axes above the figures correspond to the direction of the view. Figures 3(c1c5) show the morphologies of the grain losing the competition from different views. The competition result of the two grains is shown in the lower area of Fig. 3(a2) where the two grains make contact. The primary branches of the winning grain in the competition go through the blocking grain and continue to grow in its direction of optimal growth, as the bottom primary branch shown in Fig. 3(b2). In comparison, the primary branches of the inferior grain stop growing in the optimal growth direction and then turn to grow in areas without obstacles, as shown in Figs.
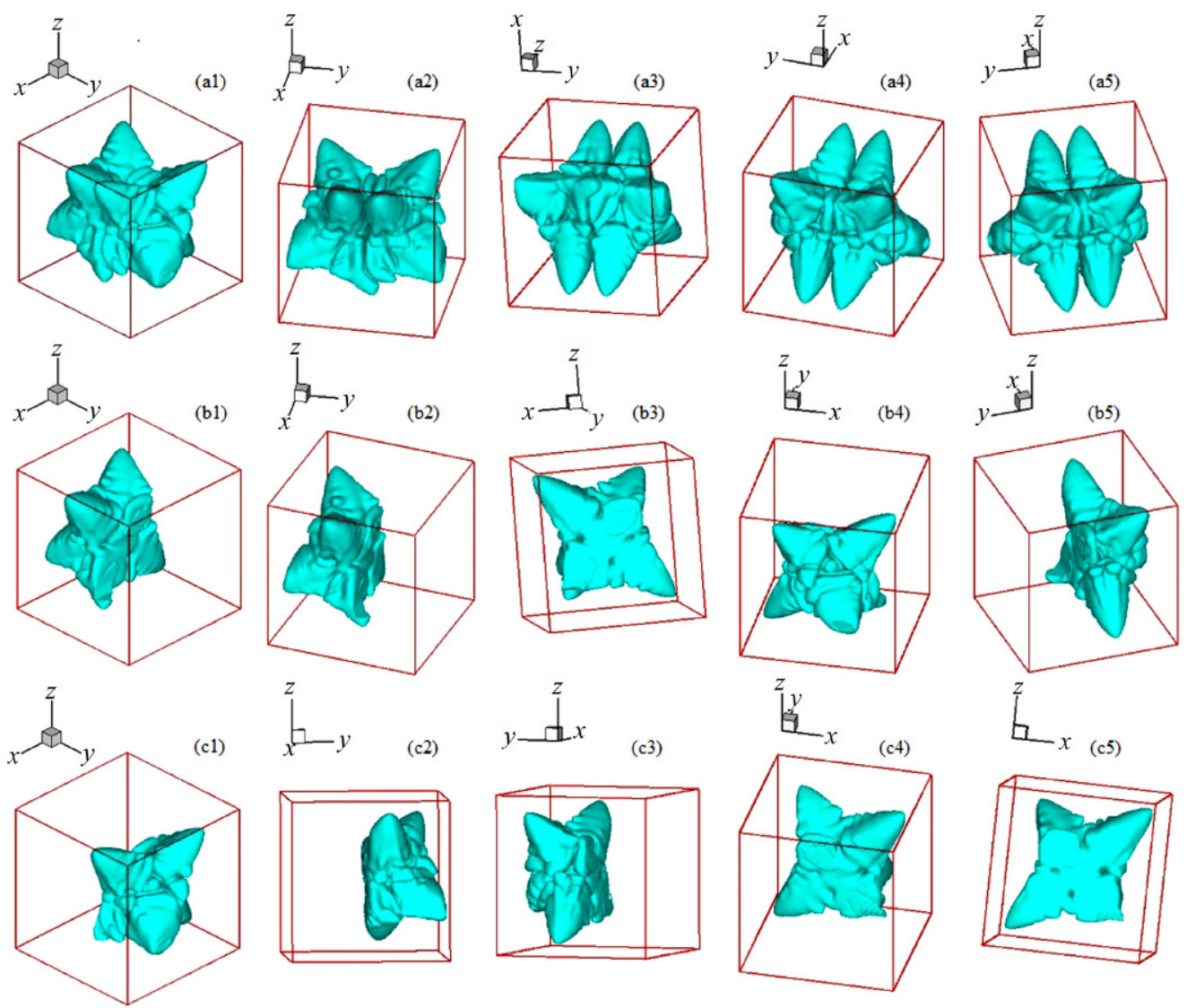

(c3)
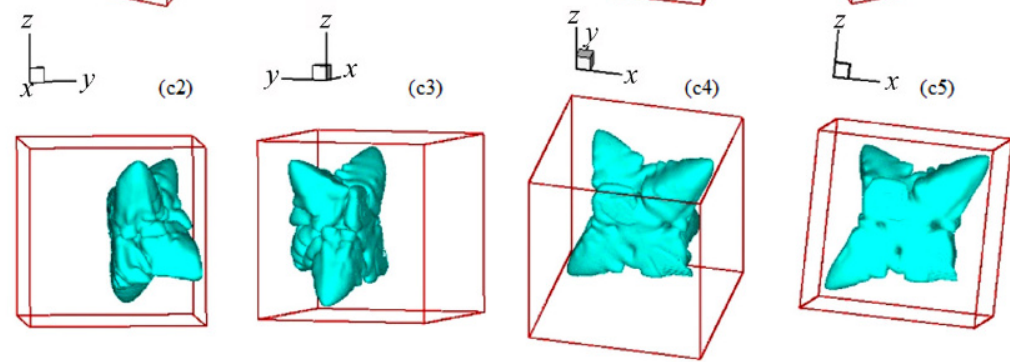

Fig. 3: Simulated morphologies of two grains with high-angle boundaries in competitive growth 
3(a2) and 3(c2). For different grains with small angles between the optimal growth directions of such dendrites, competitive growth between dendrites in Figs. 2(a2) and 2(a4) is not observed.

Classic theory suggests that the section of a dendrite tip is parabolic upon isothermal solidification ${ }^{[37,38]}$. Ivantsov derived an equation for calculating the dendrite tip velocity and constructing the shape curve of the dendrite tip ${ }^{[39]}$. By observing the grain morphologies in Figs. 1(b7) and 3, it can be seen that morphologies of the primary branches not participating in competitive growth conform to classical theory. Morphologies of the primary branches of grains taking part in the competition present different morphological features for the two types of competitive growth mentioned earlier.

The dendritic morphology of the first type of competitive growth shows the following features: a plane is formed between the dendrite tip and the competitive dendrite which prevents its normal growth in the area under competition. Both dendrites in the competition stop growing in the direction perpendicular to this plane, while they begin to compete for growth in the direction parallel to this plane. The primary branches of the grains which take part in the competition stop growing in their optimal growth directions when the optimal growth directions form a small angle. As a result, the competition plane enlarges, namely, neither one wins the competition, which can be observed from the competitive growth of grains in Figs. 2(a2) and 2(a4). When the optimal growth directions of the primary branches of grains taking part in the competition form a large angle with the optimal direction, the primary branches stop growing before one wins the competition. Also, the competition plane extends [see the competitive growth of grains in Figs. 2(a3) and 2(a5)]. When the competitive primary branches of the grains form a large angle with the optimal growth direction and win the competition, the primary branches of the superior grain cross the blocking grain and continue to grow in their optimal growth direction. The primary branches of the inferior grain stop growing in the optimal growth direction and then turn to grow in areas without obstacles, as shown in Figs. 3(b3) and 3(c5).

The dendritic morphology of second type of competitive growth shows the following features: the morphology of primary branches of different grains is deformed due to the influence of the primary branches of the competing grains. The deformation is expressed as a deflection or bending as observed from different views.

\subsection{Experimental results}

The $\mathrm{Al}-2 \%$ moleCu binary alloy was melted in an intermediate frequency induction furnace, and the sample was prepared by metal mold casting. Metallographic images are shown in Fig 4.

In Fig. 4, the competitive growths between grains $\mathrm{A}$ and $\mathrm{B}$ and grains $\mathrm{F}$ and $\mathrm{G}$ include two cases of the first type of competitive growth, as described above, which are the competitive growth of grains in Fig. 1(b7) (two-dimensional plane) and Figs. 2(a2) and 2(a4) (three-dimensional morphology), the competitive growth of grains in Fig. 1(b5) (two-dimensional plane) and Figs. 2(a3) and 2(a5) (three-dimensional morphology), respectively. The competitive growths between grains $\mathrm{C}$ and $\mathrm{D}$, and grains $\mathrm{C}$ and $\mathrm{E}$, belong to the second type of competitive growth, as described above in Figs. 2(a3) and 2(a4) (three-dimensional morphology).

Through the metallographic picture, the morphology of the competitive growth can be seen, but it is a two-dimensional plane which is just seen from a single direction, while the simulation method can show the morphology of the competitive growth in all directions. Therefore, the simulation method can better characterize the competitive growth process.

\section{Conclusions}

(1) The 3-D competitive growth of multiple dendrites is simulated to reproduce the competitive growth of equiaxed dendrites in different directions using PFM and MPI parallel computing. The competitive growth of multiple grains found in the simulations agreed well with the experimental results.

(2) The competitive growth of equiaxed dendrites can be divided into two types: the first one, the tips of competitive primary branches of different dendrites stop growing in their optimal growth direction; and the second one, the tips of competitive primary branches of different dendrites do not stop growing in their optimal growth direction during competitive growth.

(3) The morphology of the dendrites during their growth displays the following characteristics: the dendrites showing the first type of competitive growth can be divided into two cases. The competitive primary branches of dendrites stop growing in their optimal growth direction and the competitive growth plane enlarges when neither one wins the competition. The primary branches of the dendrite winning the competition cross the blocking one and continue to grow in the direction of
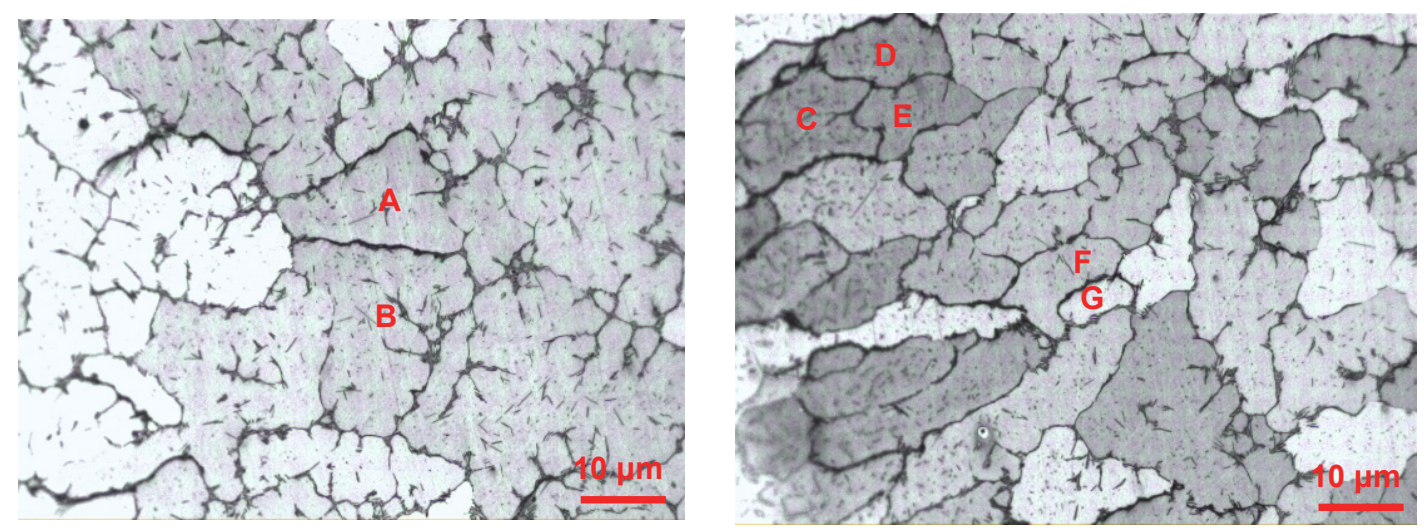

Fig. 4: Microstructure of $\mathrm{Al}-2 \%$ moleCu binary alloy 
optimal growth. The primary branches of the dendrite losing the competition stop growing in their optimal growth direction and then they turn to grow in areas without obstacles. The morphology of primary branches of the dendrite in the second type of competition is deformed through deflection and bending as observed from different views.

(4) Compared with the metallographic picture, the simulation results can show the morphology of the competitive growth in all directions, so the simulation method can better characterize the competitive growth process.

\section{References}

[1] Shibuta Y, Ohno M, Takaki T. Solidification in a supercomputer: From crystal nuclei to dendrite assemblages. JOM, 2015, 67(8): 1793-1804.

[2] Yang M, Xiong S M, Guo Z. Characterisation of the 3-D dendrite morphology of magnesium alloys using synchrotron $\mathrm{X}$-ray tomography and 3-D phase-field modelling. Acta Materialia, 2015, 92: 8-17.

[3] Nestler B, Danilov D, Bracchi A, et al. A metallic glass composite: Phase-field simulations and experimental analysis of microstructure evolution. Materials Science and Engineering A, 2007, 452-453: 8-14.

[4] Hong Wei, Wang Xiao. A phase-field model for systems with coupled large deformation and mass transport. Journal of the Mechanics and Physics of Solids, 2013, 61: 1281-1294.

[5] Gong X B, Chou K. Phase-Field Modeling of Microstructure Evolution in Electron Beam Additive Manufacturing. JOM, 2015, 67(5): 1176-1182.

[6] Kundin J, Mushongera L, Emmerich $\mathrm{H}$. Phase-field modeling of microstructure formation during rapid solidification in Inconel 718 superalloy. Acta Materialia, 2015, 95: 343-356.

[7] Ohno M, Maruyama M, Matsuura K. Experimental Verification of a Critical Condition for the Formation of As-Cast Coarse Columnar Austenite Grain Structure in a Hyperperitectic Carbon Steel. Metallurgical and Materials Transactions A, 2015, 46A (11): 5240-5247.

[8] Steinmetz P, Yabansu Y C, Hoetzer J, et al. Analytics for microstructure datasets produced by phase-field simulations. Acta Materialia, 2016, 103: 192-203.

[9] Nestler B, Danilov D, Galenko P. Crystal growth of pure substances: Phase-field simulations in comparison with analytical and experimental results. Journal of Computational Physics, 2005, 207(1): 221-239.

[10] Karma A, Rappel W J. Phase-field Method for Computationally Efficient Modeling of Solidification with Arbitrary Interface Kinetics. Physical Review E, 1996, 53(4): 3017-3020.

[11] Yuan Xunfeng, Liu Baoying, Li Chun, et al. Phase field model for strong interfacial energy anisotropy of HCP materials. Trans. Nonferrous Met. Soc. China, 2014, 24: 2911-2919.

[12] Kim S G, Kim W T, Suzuki T. Interfacial Compositions of Solid and Liquid in a Phase-field Model with Finite Interface Thickness for Isothermal Solidification in Binary Alloys. Physical Review E, 1998, 58(3): 3316-3323

[13] Kim S G, Kim W T, Lee J S, et al. Large-Scale Simulation of Dendritic Growth in Pure Undercooled Melt by Phase-field Model. ISIJ INT, 1999, 39 (4): 335-340.

[14] Kim S G, Kim W T, Suzuki T. Phase-field Model for Binary Alloys. Physical Review E, 1999, 60(6): 7186-7197.

[15] Ode M, Suzuki T, Kim S G, et al. Phase-field model for Solidification of Fe-C alloys. Science and Technology of Advanced Materials, 2000 (1): 43-49.

[16] Ode M, Lee J S, Kim S G, et al. Phase-field Model for Solidification of Ternary Alloys. ISIJ INT, 2000, 40(9): 870-876.

[17] Zhang Ruijie, Jing Tao, Jie Wanqi, et al. Phase-field Simulation of Solidification in Multicomponent Alloys Coupled with Thermodynamic and Diffusion Mobility Databases. Acta Materialia, 2006, 54: 2235-2240.
[18] Zhang Shuzhou, Zhang Ruijie, Qu Xuanhui, et al. Phase field simulation for non-isothermal solidification of multicomponent alloys coupled with thermodynamics database. Trans. Nonferrous Met. Soc. China, 2013, 23: 2361-2367.

[19] Karma A, Rappel W J. Numerical Simulation of Three-dimensional Dendritic Growth. Physical Review Letter, 1996, 77(10): 4050-4053.

[20] Zhu Changsheng, Jia Jinfang, Feng Li, et al. Research of threedimensional dendritic growth using phase-field method based on GPU. Computational Materials Science, 2014, 91: 146-152.

[21] Haxhimali T, Karma A, Gonzales F, et al. Orientation selection in dendritic evolution. Nature Materials, 2006(5): 660-664.

[22] Bragard J, Karma A, Lee Y H, et al. Linking Phase-Field and Atomistic Simulations to Model Dendritic Solidification in Highly Undercooled Melts. Interface Science, 2002 (10): 121-136

[23] Pusztai T, Bortel G and Granasy L. Phase field theory of polycrystalline solidification in three dimensions. Europhysics Letters, 2005, 71: 131-137

[24] Wesner E, Choudhury A, August A, et al. A phase-field study of largescale dendrite fragmentation in Al-Cu. Journal of Crystal Growth, 2012, 359: 107-121.

[25] Bollada P C, Goodyer C E, Jimack P K, et al. Three dimensional thermal-solute phase field simulation of binary alloy solidification. Journal of Computational Physics, 2015, 287: 130-150

[26] Yang M, Xiong S M, Guo Z. Effect of different solute additions on dendrite morphology and orientation selection in cast binary magnesium alloys. Acta Materialia, 2016, 112: 261-272.

[27] Gaubert A, Jouiad M, Cormier J, et al. Three-dimensional imaging and phase-field simulations of the microstructure evolution during creep tests of $<011>$-oriented Ni-based superalloys. Acta Materialia, 2015, 84: 237-255.

[28] Feng Li, Wang Zhiping, Zhu Changsheng, et al. Phase-field model of isothermal solidification with multiple grains growth. Chinese Physics B, 2009, 18(05): 1985-1990.

[29] Feng Li, Wang Zhiping, Lu Yang, et al. Phase-field model of isothermal solidification of binary alloy with multiple grains. Acta Physica Sinica, 2008, 57(02): 1084-1090.

[30] Li J J, Wang J C, Xu Q, et al. Comparison of Johnson-Mehl-AvramiKologoromov (JMAK) kinetics with a phase field simulation for polycrystalline solidification. Acta Materialia, 2007, 55: 825-832.

[31] Takaki T, Shimokawabe T, Ohno M, et al. Unexpected selection of growing dendrites by very-large-scale phase-field simulation. Journal of Crystal Growth, 2013, 382: 21-25.

[32] Souhar Y, De Felice V F, Beckermann C, et al. Three-dimensional mesoscopic modeling of equiaxed dendritic solidification of a binary alloy. Computational Materials Science, 2016, 112: 304-317.

[33] Takaki T, Ohno M, Shimokawabe T, et al. Two-dimensional phasefield simulations of dendrite competitive growth during the directional solidification of a binary alloy bicrystal. Acta Materialia, 2014, 81: 272-283.

[34] Takaki T, Ohno M, Shibuta Y, et al. Two-dimensional phase-field study of competitive grain growth during directional solidification of polycrystalline binary alloy. Journal of Crystal Growth, 2016, 442: 14-24.

[35] Tourret D, Karma A. Growth competition of columnar dendritic grains: A phase-field study. Acta Materialia, 2015, 82: 64-83.

[36] Feng Li, Jia Jinfang, Zhu Changsheng, et al. Research on PhaseField Model of Three-Dimensional Dendritic Growth for Binary Alloy. Journal of Computational and Theoretical Nanoscience, 2015, 12(11): 4289-4296.

[37] Lipton J, Glicksman M E, Kurz W. Dendritic growth into undercooled alloy metals. Materials Science \& Engineering, 1984, 65(1): 57-63.

[38] Lipton J, Glicksman M E, Kurz W. Equiaxed dendrite growth in alloys at small supercooling. Metallurgical \& Materials Transactions A, 1987, 18(2): 341-345.

[39] Lipton J, Kurz W, Trivedi R. Rapid dendrite growth in undercooled alloys. Acta Metallurgica, 1987, 35(4): 957-964. 